\title{
Expression of ALDH1A1 and CD44 in primary head and neck squamous cell carcinoma and their value for carcinogenesis, tumor progression and cancer stem cell identification
}

\author{
MARTIN LEINUNG ${ }^{1}$, BENJAMIN ERNST $^{1}$, CONSTANZE DÖRING $^{2}$, JENS WAGENBLAST $^{1}$, \\ AYKUT TAHTALI $^{1}$, MARC DIENSTHUBER $^{1}$, TIMO STÖVER $^{1}$ and CHRISTIN GEISSLER ${ }^{1}$ \\ ${ }^{1}$ Department of Otolaryngology; ${ }^{2}$ Institute of Pathology, University Hospital Frankfurt, Frankfurt am Main 60590, Germany
}

Received September 23, 2014; Accepted June 2, 2015

DOI: $10.3892 / 01.2015 .3542$

\begin{abstract}
In head and neck squamous cell carcinoma (HNSCC), aldehyde dehydrogenase 1 family, member A1 (ALDH1A1) and hyaluronan receptor cluster of differentiation 44 (CD44) are often used as cancer stem cell (CSC) markers. The aim of the present study was to examine the relevance of these proteins for HNSCC in general and for the identification of CSCs. Tumors from 48 patients with primary HNSCC were analyzed for the expression of ALDH1A1 and CD44. Additionally, the association of the proteins with the proliferation rate and epidermal growth factor receptor (EGFR) expression was analyzed. ALDH1A1 was expressed in $54.2 \%$ of the carcinoma samples while CD44 was expressed in $89.6 \%$ of the carcinoma samples. Most notably, these proteins were often not expressed exclusively in a subpopulation, but also in the majority of tumor cells (ALDH1A1: $30.8 \%$ of ALDH1 A1 ${ }^{+}$tumors; CD44: $65.1 \%$ of $\mathrm{CD}_{4} 4^{+}$tumors). Furthermore, patients with $\mathrm{ALDH} 1 \mathrm{~A} 1^{+}$tumors exhibited worse survival rates. CD44 and EGFR expression patterns were overlapping within the tumors and the expression rates were significantly connected. Ki- $67^{+}$ tumor cells often expressed CD44. ALDH1A1 and CD44 expression patterns only partly overlapped. Consequently, ALDH1A1 and CD44 play significant roles in carcinogenesis and tumor progression. Within the present study, CD44 appeared to interact with EGFR and was more often expressed in primary HNSCC than the marker ALDH1A1. However, ALDH1A1 was a better marker to define a subpopulation of tumor cells. Finally, neither ALDH1A1 nor CD44, alone or combined, were sufficient to determine the CSC population in HNSCC.
\end{abstract}

Correspondence to: Mrs. Christin Geissler, Department of Otolaryngology, University Hospital Frankfurt, 7 Theodor-Stern-Kai, Building 8D, Frankfurt am Main 60590, Germany

E-mail: christin.geissler@kgu.de

Key words: CD44, ALDH1A1, cancer stem cells, head and neck squamous cell carcinoma

\section{Introduction}

Head and neck squamous cell carcinoma (HNSCC) is the most common entity of the upper aerodigestive tract (1). Carcinoma of this area is the fourth most common region of cancer incidence and the second most common region for cancer-related mortality worldwide (2). The intramural heterogeneity may be an explanation for the worse survival rates recorded in HNSCC. Cancer stem cells (CSCs) are the most important subpopulation within carcinoma. CSCs are associated with a worse response to therapy, metastasis and cancer relapse (3). Aldehyde dehydrogenase 1 family, member A1 (ALDH1A1) and hyaluronan receptor cluster of differentiation 44 (CD44) are common markers for CSCs in HNSCC (4-6).

ALDH1A1 belongs to the ALDH enzyme family and catalyzes the oxidation of aldehydes to carboxylic acids (7). This enzyme mediates protection from chemotherapy and reactive oxygen species (ROS) $(7,8)$.

CD44 is an important hyaluronan receptor. CD44 acts in cell aggregation, proliferation and migration (9). In HNSCC, CD44 is associated with tumor invasion (10) and the poor survival of tumor patients (11).

The present study analyzed the expression of ALDH1A1 and CD44 for their relevance in human primary HNSCC. The prognostic value of ALDH1A1 expression was estimated. Moreover, the utility of ALDH1A1 and CD44 was evaluated in order to identify CSCs. To define the CSC population and its activity more precisely, features such as proliferation (Ki-67) and growth receptor expression [epidermal growth factor receptor (EGFR)] were included into the analysis.

\section{Materials and methods}

Patients. In this study, 48 patients with primary HNSCC (35 male and 13 female) were investigated. Tumor samples were collected between 1997 and 2008. Experiments were approved by the Ethics Committee of the Department of Medicine of the Johann Wolfgang Goethe-University, Frankfurt am Main (276/12).

Sample preparation and analysis. All available samples of each primary tumor were examined. For immunohistochemistry, 
Table I. ALDH1A1 and/or CD44 expression in human primary tumors. ${ }^{a}$

\begin{tabular}{|c|c|c|c|c|}
\hline \multirow[b]{2}{*}{ Expression } & \multicolumn{4}{|c|}{ ALDH1A1 and/or CD44 expression, n (\%) } \\
\hline & $\mathrm{ALDH}_{1 \mathrm{~A}} 1^{+}$ & $\mathrm{CD} 44^{+}$ & $\mathrm{ALDH} 1 \mathrm{~A} 1^{+} / \mathrm{CD} 44^{+}$ & ALDH1A1\%CD44 \\
\hline General expression & $26(54.2)$ & $43(89.6)$ & $24(50.0)$ & $3(6.3)$ \\
\hline Majority ALDH1A1+ & $8(30.8)$ & $8(18.6)$ & $8(33.3)$ & - \\
\hline Minority ALDH1A1+ & $18(69.2)$ & $16(37.2)$ & $16(66.7)$ & - \\
\hline ALDH1A1- & - & $19(44.2)$ & - & $3(100.0)$ \\
\hline Majority CD $44^{+}$ & $13(50.0)$ & $28(65.1)$ & $11(45.8)$ & - \\
\hline Minority CD $44^{+}$ & $11(42.3)$ & $15(34.9)$ & $13(54.2)$ & - \\
\hline CD44- & $2(7.7)$ & - & - & $3(100.0)$ \\
\hline
\end{tabular}

${ }^{a} \mathrm{CD} 44$ and/or ALDH1A1 were expressed in $93.8 \%$ of carcinoma samples. CD44 was more often expressed, but ALDH1A1 more frequently represented a minority of tumor cells. ALDH1A1, aldehyde dehydrogenase 1 family, member A1; CD44, hyaluronan receptor cluster of differentiation 44 .

Table II. Staining intensity of CD44 and EGFR in human primary tumors. Linear regression of expression (P $\leq 0.01)$.

\begin{tabular}{lccr}
\hline & \multicolumn{2}{c}{ Staining intensity of CD44 and EGFR, $(\%)$} \\
\cline { 2 - 4 } Protein & - & + & ++ \\
\hline EGFR & $1(2.1)$ & $12(25.0)$ & $9(18.8)$ \\
CD44 & $5(10.4)$ & $5(10.4)$ & $14(29.2)$
\end{tabular}

ALDH1A1, aldehyde dehydrogenase 1 family, member A1; CD44, hyaluronan receptor cluster of differentiation 44.

formaldehyde-fixed paraffin-embedded tumor samples, cut to a size of $5 \mu \mathrm{m}$, were used. Antigen retrieval was performed in boiling citrate buffer (pH 6.0; S1699; Dako, Glostrup, Denmark). ALDH1A1 and CD44 are common markers for the identification of CSC (4-6), therefore, these markers were used to identify tumor cell subpopulations comprised of CSCs. The expression of anti-human rabbit monoclonal ALDH1A1 (1/100, ab52492; Abcam, Cambridge, UK), anti-human mouse monoclonal CD44 (1/100, MU310-UC; BioGenex, Fremont, CA, USA), anti-human mouse monoclonal EGFR (1/50, ab49716; Abcam) and anti-human rabbit monoclonal Ki-67 (1/200, KI68IC01: DCS, Hamburg, Germany) were detected. The samples were incubated with primary antibody for $1 \mathrm{~h}$ at room temperature. In the next step of immunohistochemistry staining procedure, the DCS Detection Line system staining kits (AD050POL-K, PD000RP and DD006RAP; DCS, Hamburg, Germany) were used. Staining was developed with DAB reagent (DC137C100; DCS) and a Fuchsin-Substrate-Chromogen System (K0625; Dako). Images were captured with a Zeiss Axioplan2 (AxioCam ICc1 camera; Zeiss, Oberkochen, Germany). The staining intensity of CD44 and EGFR in the tumor cells was scaled as follows: Strong $(+++)$, moderate $(++)$, weak $(+)$ and none $(-)$. Weak staining was described as less intensive and/or discontinuous membrane staining of the tumor cells.

So far as documented, the clinical history of the patients and the tumor-node-metastasis (TNM) status (12) of the carcinoma were analyzed.
Statistical analysis. The statistical analysis of data was performed with BiAS software for Windows (version 10.12; Epsilon-Verlag, Frankfurt, Germany). The significance between CD44 and EGFR was analyzed by Yates-Cochram test of trends. The significance of cancer relapse for the ALDH1A1 expression groups (+/-) was analyzed by Fisher's exact test. $\mathrm{P}<0.05$ was considered to indicate a statistically significant difference.

\section{Results}

General ALDHIAl and CD44 expression. The majority of primary HNSCC tumors $(45 / 48 ; 93.8 \%)$ expressed a minimum of one protein (Table I). Only three samples (6.3\%) expressed neither ALDH1A1 nor CD44. CD44 expression was detected in $89.6 \%$ (43/48) of studied tumors. CD44 expression intensity was scaled within the tumor cells (Table II). In $55.8 \%$ (24/43) of the $\mathrm{CD}_{4} 4^{+}$tumors expression for the receptor was strong $(+++)$, in $32.6 \%(14 / 43)$ the CD44 expression was moderate $(++)$ and in $11.6 \%(5 / 43)$ of samples the CD44 level was weak (+). In $65.1 \%(28 / 43)$ of the $\mathrm{CD}_{4} 4^{+}$tumors this receptor was expressed in almost all tumor cells. The majority of CD44 tumors $(38 / 43,88.4 \%)$ expressed this receptor at a strong to moderate level $(++/+++)$. Tumor cell nests were solely CD $44^{+}$ or CD44-expressing tumor cells were located in the periphery of the tumor cell nests and close to the stroma (Fig. 1D).

ALDH1A1 expression was detected in 54.2\% (26/48) of the studied tumor samples (Table I). In $30.8 \%(8 / 26)$ of the 

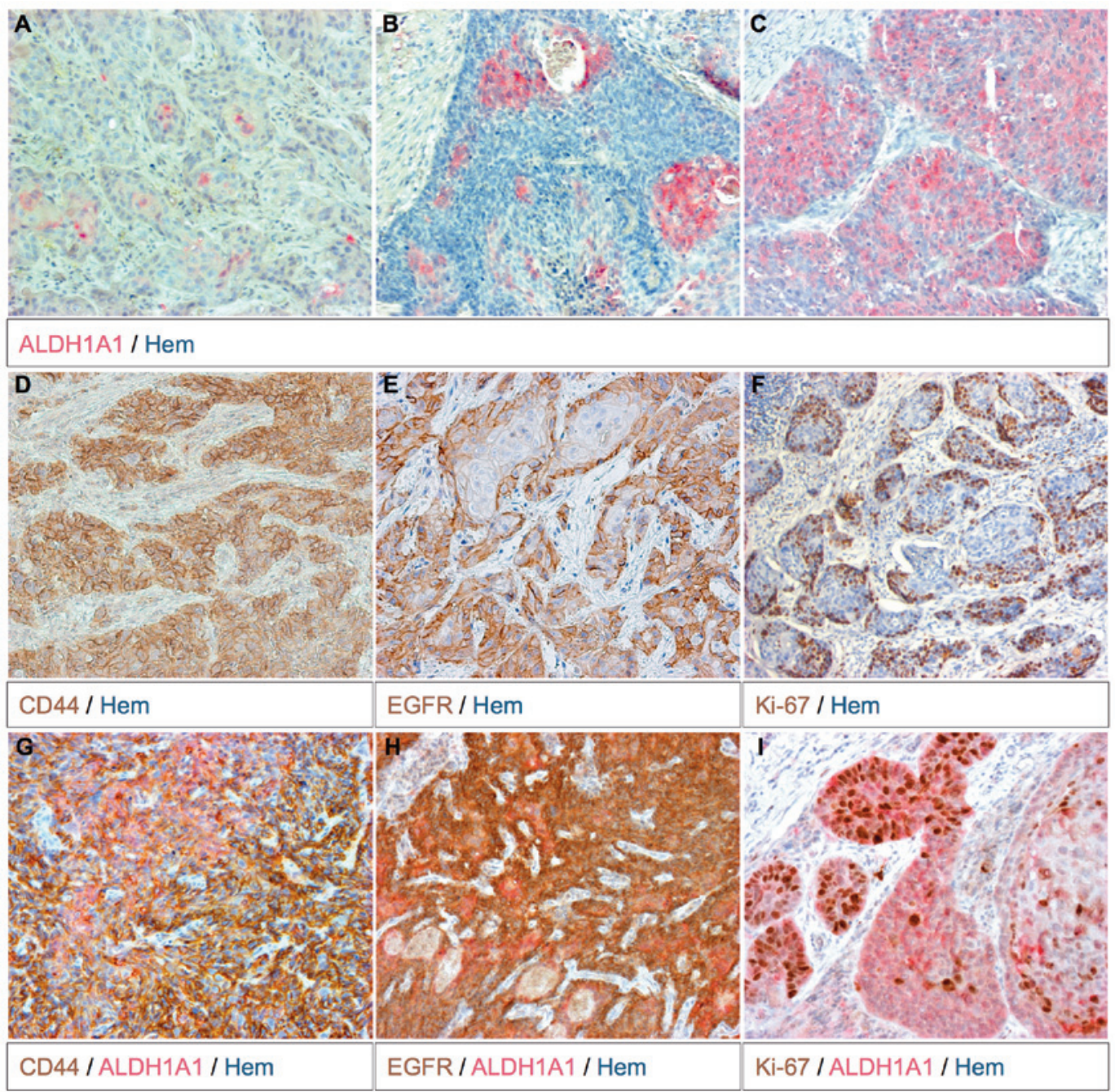

Figure 1. Immunohistochemistry of human primary tumors. (A and B) ALDH1 A1 ${ }^{+}$cells were located singularly, in groups and more centrally within cell nests . (C) Tumor expressing ALDH1 A1 completely. (D-F) CD44, EGFR and Ki-67 were expressed marginally to generally within the cell nests. (G and H) ALDH1A1 expression pattern only partly overlapped with CD44 or EGFR. (I) The rate of Ki-67+ cells was independent from ALDH1A1 expression. Magnification, x200; hem counterstaining. ALDH1A1, aldehyde dehydrogenase 1 family, member A1; CD44, hyaluronan receptor cluster of differentiation 44; EGFR, epidermal growth factor receptor; hem, hematoxylin.

ALDH1A1 $1^{+}$tumors this enzyme was expressed in the majority of tumor cells (Fig. 1C). In the remaining ALDH1A1 $1^{+}$tumors $(18 / 26,69.2 \%)$ ALDH1A1-expressing cells were found to be singular, in groups or throughout the entirely cell nest (Fig. 1A and $\mathrm{B})$. More often ALDH1A1+ tumor cell groups were located more centrally within the cell nests (Fig. 1A and B).

Half (24/48) of the analyzed tumors expressed ALDH1A1 and CD44 (Table I). In these tumors the expression pattern of ALDH1A1 and CD44 intersected, but did not completely overlap (Fig. 1G). With the exception of one tumor $(7 / 8$, $87.5 \%$ ), the human papilloma virus $(\mathrm{HPV})^{+}$tumors expressed ALDH1A1 and CD44. The remaining $\mathrm{HPV}^{+}$tumor showed CD44 staining (Table III).

EGFR and Ki67 status of ALDH1A1 ${ }^{+}$or $\mathrm{CD}_{4} 4^{+}$tumor cells. With the exception of one tumor, the examined HNSCC tumors expressed EGFR (47/48, 97.9\%) (Table II). In most tumors the majority of tumor cells were EGFR ${ }^{+}$. The staining intensity of EGFR was scaled as strong to moderate $(++/+++)$ in $74.5 \%$ (35/47) of EGFR ${ }^{+}$tumors. The staining patterns of CD44 and
EGFR were mostly overlapping. The position of the $\mathrm{EGFR}^{+}$and $\mathrm{CD}_{4} 4^{+}$tumor cells was generally identical. However, EGFR ${ }^{+}$ tumor cells were located more marginally within the tumor cell nests (Fig. 1E). The association between EGFR and CD44 staining intensity could be described as an linear regression $(\mathrm{P} \leq 0.01)$. In the majority of tumors, ALDH1A1 $1^{+}$tumor cells were not necessarily CD44+ ${ }^{+}$(Fig. 1G). EGFR staining gave similar results (Fig. $1 \mathrm{H}$ ). The amount of $\mathrm{Ki}-67^{+}$tumor cells also appeared to be independent of ALDH1A1 expression (Fig. 1I). Closer to the stroma the proliferation rate was higher (Fig. 1F).

Analysis of $\mathrm{ALDHIA1}^{+}$and $\mathrm{ALDHIA1}^{-}$patients. The patient collective was divided into ALDH1A1 ${ }^{+}$and ALDH1A1 primary tumor samples. Notably, the majority of confirmed tonsillar tumors $(11 / 16 ; 68.8 \%)$ were $\mathrm{ALDH} 1 \mathrm{~A} 1^{+}$. Due to the low number of CD44- tumors $(5 / 48 ; 10.4 \%)$, an analysis of $\mathrm{CD}_{4}{ }^{+}$and CD44- samples was not meaningful.

The appearance of metastasis and relapse was analyzed (Table III). In the majority of cases, cancer relapse was 
Table III. Evaluation of available patient data for ALDH1A1 expression and HPV status. ${ }^{\mathrm{a}}$

\begin{tabular}{|c|c|c|c|c|}
\hline \multirow[b]{2}{*}{ Factor } & \multicolumn{4}{|c|}{ Evaluation of patient data, n (\%) } \\
\hline & $\mathrm{ALDH} 1 \mathrm{~A} 1^{+}$ & $\mathrm{ALDH} \mathrm{A}^{+} / \mathrm{HPV}^{-}$ & ALDH1A1 ${ }^{-}$ & ALDH1A1\%/HPV \\
\hline Total patients & $26(100.0)$ & $19(100.0)$ & $22(100.0)$ & $21(100.0)$ \\
\hline \multicolumn{5}{|l|}{ Gender } \\
\hline Male & 20 (76.9) & $14(73.7)$ & $15(68.2)$ & $15(71.4)$ \\
\hline Female & $6(23.1)$ & $5(26.3)$ & $7(31.8)$ & $7(33.3)$ \\
\hline \multicolumn{5}{|l|}{ Cancer relapse } \\
\hline Positive & $6(23.1)$ & $6(31.6)$ & $12(54.5)$ & $12(57.1)$ \\
\hline Negative & $20(76.9)$ & $13(68.4)$ & $10(45.5)$ & $9(42.9)$ \\
\hline \multicolumn{5}{|l|}{ Age, years } \\
\hline$<60$ & $10(38.5)$ & $7(36.8)$ & $13(59.1)$ & $12(57.1)$ \\
\hline $60-70$ & $9(34.6)$ & $9(47.4)$ & $7(31.8)$ & $7(33.3)$ \\
\hline$>70$ & 7 (26.9) & $3(15.8)$ & $2(9.1)$ & $2(9.5)$ \\
\hline T stage & $25(100.0)$ & $18(100.0)$ & $18(100.0)$ & $17(100.0)$ \\
\hline $\mathrm{T} 1$ & $0(0.0)$ & $0(0.0)$ & $4(22.2)$ & $4(23.5)$ \\
\hline $\mathrm{T} 2$ & $12(48.0)$ & $9(50.0)$ & $7(38.9)$ & $7(41.2)$ \\
\hline $\mathrm{T} 3$ & $9(36.0)$ & $5(27.8)$ & $5(27.8)$ & $4(23.5)$ \\
\hline $\mathrm{T} 4$ & $4(16.0)$ & $4(22.2)$ & $2(11.1)$ & $2(11.8)$ \\
\hline $\mathrm{N}$ stage & $24(100.0)$ & $17(100.0)$ & $18(100.0)$ & $17(100.0)$ \\
\hline No & $8(33.3)$ & $4(23.5)$ & $6(33.3)$ & $6(35.3)$ \\
\hline N1-N3 & $16(66.7)$ & $13(76.5)$ & $12(66.7)$ & $11(64.7)$ \\
\hline
\end{tabular}

Patients with ALDH1A1 ${ }^{+}$tumors exhibited a reduced risk for relapse $(\mathrm{P} \leq 0.05)$. Metastasis into lymph nodes $\left(\mathrm{N}\right.$ status) between $\mathrm{ALDH} 1 \mathrm{~A} 1^{+}$ and ALDH1A1' tumors was equal. ALDH1A1, aldehyde dehydrogenase 1 family, member A1; CD44, hyaluronan receptor cluster of differentiation 44; HPV, human papilloma virus.

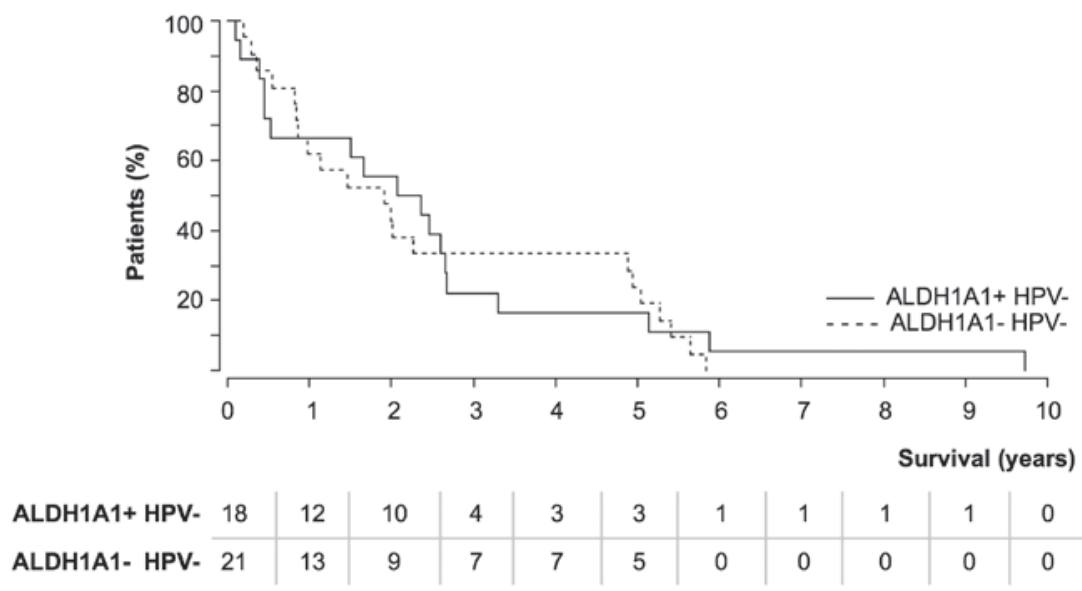

Figure 2. Survival rates of patients with ALDH1A1 $1^{+}$or ALDH1A1 tumors with known day of mortality. Patients with ALDH1A1+ tumors appeared to exhibit reduced survival. (HPV was an independent prognostic factor for better survival). ALDH1A1, aldehyde dehydrogenase 1 family, member A1; CD44, hyaluronan receptor cluster of differentiation 44; HPV, human papilloma virus.

observed up to 2 years after primary tumor detection. Cancer relapse occurred in $23.1 \%(6 / 26)$ of patients with ALDH1A1 ${ }^{+}$ primary tumors. The number of relapses was significantly higher $(\mathrm{P} \leq 0.05)$ for patients with $\mathrm{ALDH}^{\mathrm{A}} 1^{-}$primary tumors $(12 / 22 ; 54.5 \%)$. No notable differences were found between the TNM stage of ALDH1A1 ${ }^{+}$and ALDH1A1- tumors. However, one explanation for the increased number of relapses resulted in the comparison of overall survival (Fig. 2). As HPV is a prognostic factor for longer survival (13), $\mathrm{HPV}^{+}$cases were excluded.

The majority of patients in the two groups succumbed within the first 3 years of tumor appearance. Within the ALDH1A1 ${ }^{+}$ $\left(\mathrm{HPV}^{-}\right)$tumor group, fewer patients $(4 / 18 ; 22.2 \%)$ survived longer than 3 years compared with the ALDH1A1- $\left(\mathrm{HPV}^{-}\right)$ 
tumor group $(7 / 21 ; 33.3 \%)$. This issue could not be explained by a younger age of the ALDH1A1' tumor patients, as the analysis of different age groups $(<60,60-70$ and $>70$ years $)$ gave comparable results (data not shown). Survival was not linked to the percentage of $\mathrm{ALDH} 1 \mathrm{~A} 1^{+}$cells within the tumor.

\section{Discussion}

ALDH1A1 and CD44 function in the carcinogenesis and tumor progression of HNSCC. ALDH1A1 is not expressed in the normal oral mucosa $(14,15)$. However, other ALDH isoenzymes, such as ALDH1A3 and ALDH3A1, are found (14). The expression of the retinoic acid receptors in the oral mucosa underlines the function of ALDH isoenzymes in the normal mucosa (16). However, ALDH1A1 expression is increased in dysplasia and HNSCC (6). In the present study, it was found that $54.2 \%$ of primary HNSCC tumors were ALDH1 $\mathrm{A}^{+}$. In $30.8 \%$ of the ALDH1A1 ${ }^{+}$tumors, ALDH1A1 was expressed in the majority of tumor cells. This observations confirm a more general function of ALDH1A1 in the carcinogenesis of HNSCC. One explanation is that ALDH1A1 is stress-induced. The ALDH1A1 promoter has binding sites for the AP-1 (17) and Oct-1 (18) transcription factors. Oct-1 is stabilized and translocated into the nucleus due to radiation, ROS (19) and HPV16 infection (20). The low expression of ALDH1A1 has also been recognized in the normal tonsillar squamous epithelium (21). Additionally, ALDH1A1 protects from oxidative stress-induced reactive aldehydes (8). In the present study, ALDH1A1 ${ }^{+}$tumor cells were mostly located in the middle of tumor cell nests and assumed hypoxic tumor sites. Furthermore, ALDH1A1 appeared to be a prognostic factor for worse survival. This was in agreement with the results of a study by Qian et al (22). The reduced relapse rates for patients with ALDH1A1 ${ }^{+}$tumors may arise from worse survival rates. Additionally, the majority of HPV-associated carcinomas

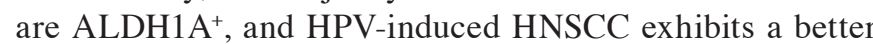
prognosis (13). These observations possibly verify the role of ALDH1A1 in tumor progression.

CD44 is expressed in the basal layer of the normal oral mucosa (21,23-25). As a consequence, CD44 was overexpressed in many of the examined tumors in the present study. CD44 was located entirely throughout the cell nest or peripheral to the stroma. CD44 can be linked to the invasion of HNSCC (10). However, the present results indicate that CD44 has a more general function in carcinogenesis. CD44 was often coexpressed with EGFR. Additionally the expression intensities of CD44 and EGFR were correlated significantly throughout the tumor. It is known that CD44 can interact with tyrosine kinase receptors, such as EGFR (26). Interaction of these surface receptors can cause proliferation, chemotherapy resistance and invasion in HNSCC cell lines (27,28). EGFR also increases CD44 mRNA expression (29). Additionally, proliferating tumor cells (Ki-67) are often $\mathrm{CD}_{4} 4^{+}$. Consequently, CD44 also has a significant role in HNSCC tumor progression.

ALDH1A1 and CD44 are common CSC markers in HNSCC (4-6). The present study examined the ability of the two proteins to identify CSCs. Corresponding to the hierarchical tumor model, CSCs exist in every tumor $(30,31)$. In the present study, the ALDH1A1 and CD44 proteins were expressed in the majority of tumors and also in $\mathrm{HPV}^{+}$ carcinoma. Consequently, ALDH1A1 and CD44 could also be expressed in the CSCs of these tumors. As a CSC marker, CD44 was more often expressed in the tumors than ALDH1A1. However, a lot of tumors expressed CD44 in almost all tumor cells. To identify CSCs, the markers must isolate CSCs from the tumor bulk. ALDH1A1 was a better marker to define a subpopulation of tumor cells. Finally, the two markers were not sufficient to isolate the CSCs from the bulk of tumor cells. Further CSC markers should be used to define and isolate the CSC population.

In addition, ALDH1A1 und CD44 expression did not completely overlap. In the majority of tumors $\mathrm{ALDH} 1 \mathrm{~A} 1^{+} / \mathrm{CD} 44^{+}, \mathrm{ALDH} 1 \mathrm{~A} 1^{+} / \mathrm{CD} 44^{-}$and $\mathrm{ALDH} 1 \mathrm{~A} 1 \% \mathrm{CD} 44^{+}$populations were observed. This observation may indicate that different CSC populations could exist within one tumor. For HNSCC, this theory of different CSC phenotypes was first suggested by Biddle et al (32). This hypothesis has also been discussed for breast $(33,34)$ and colorectal (30) cancer. Different phenotypes would make it more difficult to determine the CSC population.

In conclusion, CD44 and ALDH1A1 appear to be important factors in carcinogenesis and tumor progression. ALDH1A1 was shown to be a possible prognostic marker for worse survival, while ALDH1A1 and CD44 may be expressed in the CSCs of most examined tumors. However, these markers are not sufficient to precisely isolate the CSC subpopulation from the tumor bulk.

\section{Acknowledgements}

This study was funded by a young investigator grant of UCT Frankfurt/Main and the Heinrich and Erna Schaufler-Stiftung (grant no. SKHT-HNO-01/13).

\section{References}

1. Leemans CR, Braakhuis BJ and Brakenhoff RH: The molecular biology of head and neck cancer. Nat Rev Cancer 11: 9-22, 2011.

2. Ferlay J, Soerjomataram I, Ervik M, Dikshit R, Eser S, Mathers C, Rebelo M, Parkin DM, Forman D and Bray F: GLOBOCAN 2012, cancer incidence and mortality worldwide: IARC cancerbase No. 11 [Internet]. Lyon, France, IARC v1.0, 2013.

3. Wu MJ, Jan CI, Tsay YG, Yu YH, Huang CY, Lin SC, Liu CJ, Chen YS, Lo JF and Yu CC: Elimination of head and neck cancer initiating cells through targeting glucose regulated protein78 signaling. Mol Cancer 9: 283, 2010.

4. Chen YC, Chen YW, Hsu HS, Tseng LM, Huang PI, Lu KH, Chen DT, Tai LK, Yung MC, Chang SC, et al: Aldehyde dehydrogenase 1 is a putative marker for cancer stem cells in head and neck squamous cancer. Biochem Biophys Res Commun 385: 307-313, 2009.

5. Clay MR, Tabor M, Owen JH, Carey TE, Bradford CR, Wolf GT, Wicha MS and Prince ME: Single-marker identification of head and neck squamous cell carcinoma cancer stem cells with aldehyde dehydrogenase. Head Neck 32: 1195-1201, 2010.

6. Visus C, Ito D, Amoscato A, Maciejewska-Franczak M, Abdelsalem A, Dhir R, Shin DM, Donnenberg VS, Whiteside TL and DeLeo AB: Identification of human aldehyde dehydrogenase 1 family member A1 as a novel CD8+ T-cell-defined tumor antigen in squamous cell carcinoma of the head and neck. Cancer Res 67: 10538-10545, 2007.

7. Ma I and Allan AL: The role of human aldehyde dehydrogenase in normal and cancer stem cells. Stem Cell Rev 7: 292-306, 2011.

8. Singh S, Brocker C, Koppaka V, Chen Y, Jackson BC, Matsumoto A, Thompson DC and Vasiliou V: Aldehyde dehydrogenases in cellular responses to oxidative/electrophilic stress. Free Radic Biol Med 56: 89-101, 2013. 
9. Turley EA, Noble PW and Bourguignon LY: Signaling properties of hyaluronan receptors. J Biol Chem 277: 4589-4592, 2002.

10. Wang SJ and Bourguignon LY: Role of hyaluronan-mediated CD44 signaling in head and neck squamous cell carcinoma progression and chemoresistance. Am J Pathol 178: 956-963, 2011

11. Kokko LL, Hurme S, Maula SM, Alanen K, Grénman R, Kinnunen I and Ventelä S: Significance of site-specific prognosis of cancer stem cell marker CD44 in head and neck squamous-cell carcinoma. Oral Oncol 47: 510-516, 2011.

12. Edge SB and Compton CC: The American Joint Committee on Cancer: The 7th edition of the AJCC cancer staging manual and the future of TNM. Ann Surg Oncol 17: 1471-1474, 2010.

13. Gillespie MB, Rubinchik S, Hoel B and Sutkowski N: Human papillomavirus and oropharyngeal cancer: What you need to know in 2009. Curr Treat Options Oncol 10: 296-307, 2009.

14. Hedberg JJ, Grafström RC, Vondracek M, Sarang Z, Wärngård L and Höög JO: Micro-array chip analysis of carbonyl-metabolising enzymes in normal, immortalised and malignant human oral keratinocytes. Cell Mol Life Sci 58: 1719-1726, 2001.

15. Kato H, Izumi K, Saito T, Ohnuki H, Terada M, Kawano Y, Nozawa-Inoue K, Saito C and Maeda T: Distinct expression patterns and roles of aldehyde dehydrogenases in normal oral mucosa keratinocytes: Differential inhibitory effects of a pharmacological inhibitor and RNAi-mediated knockdown on cellular phenotype and epithelial morphology. Histochem Cell Biol 139: 847-862, 2013.

16. Sherman JA and Partridge M: Expression of retinoic acid receptors in normal, dysplastic and malignant oral epithelia. Br J Oral Maxillofac Surg 35: 260-266, 1997.

17. Makia NL, Amunom I, Falkner KC, Conklin DJ, Surapureddi S, Goldstein JA and Prough RA: Activator protein-1 regulation of murine aldehyde dehydrogenase 1a1. Mol Pharmacol 82: 601-613, 2012.

18. Maddox J, Shakya A, South S, Shelton D, Andersen JN, Chidester S, Kang J, Gligorich KM, Jones DA, Spangrude GJ, et al: Transcription factor Oct1 is a somatic and cancer stem cell determinant. PLoS Genet 8: e1003048, 2012.

19. Wang P and Jin T: Oct-1 functions as a sensor for metabolic and stress signals. Islets 2: 46-48, 2010.

20. Wong HK and Ziff EB: The human papillomavirus type $16 \mathrm{E} 7$ protein complements adenovirus type $5 \mathrm{E} 1 \mathrm{~A}$ amino-terminus-dependent transactivation of adenovirus type 5 early genes and increases ATF and Oct-1 DNA binding activity. J Virol 70: 332-340, 1996.

21. Uhlen M, Oksvold P, Fagerberg L, Lundberg E, Jonasson K, Forsberg M, Zwahlen M, Kampf C, Wester K, Hober S, et al: Towards a knowledge-based Human Protein Atlas. Nat Biotechnol 28: 1248-1250, 2010. Available from: $\mathrm{http} / / / \mathrm{www} \cdot$ proteinatlas.org
22. Qian X, Wagner S, Ma C, Coordes A, Gekeler J, Klussmann JP, Hummel M, Kaufmann AM and Albers AE: Prognostic significance of ALDH1A1-positive cancer stem cells in patients with locally advanced, metastasized head and neck squamous cell carcinoma. J Cancer Res Clin Oncol 140: 1151-1158, 2014.

23. Mack B and Gires O: CD44 s and CD44v6 expression in head and neck epithelia. PLoS One 3: e3360, 2008.

24. Sterz CM, Kulle C, Dakic B, Makarova G, Böttcher MC, Bette M, Werner JA and Mandic R: A basal-cell-like compartment in head and neck squamous cell carcinomas represents the invasive front of the tumor and is expressing MMP-9. Oral Oncol 46: 116-122, 2010.

25. Richard V and Pillai MR: The stem cell code in oral epithelial tumorigenesis: 'the cancer stem cell shift hypothesis'. Biochim Biophys Acta 1806: 146-162, 2010.

26. Williams K, Motiani K, Giridhar PV and Kasper S: CD44 integrates signaling in normal stem cell, cancer stem cell and (pre) metastatic niches. Exp Biol Med 238: 324-338, 2013.

27. Wang SJ and Bourguignon LY: Hyaluronan and the interaction between CD44 and epidermal growth factor receptor in oncogenic signaling and chemotherapy resistance in head and neck cancer. Arch Otolaryngol Head Neck Surg 132: 771-778, 2006.

28. Bourguignon LY, Gilad E, Brightman A, Diedrich F and Singleton P: Hyaluronan-CD44 interaction with leukemia-associated RhoGEF and epidermal growth factor receptor promotes $\mathrm{Rho} / \mathrm{Ras}$ co-activation, phospholipase $\mathrm{C}$ epsilon-Ca2+ signaling and cytoskeleton modification in head and neck squamous cell carcinoma cells. J Biol Chem 281: 14026-14040, 2006.

29. Abhold EL, Kiang A, Rahimy E, Kuo SZ, Wang-Rodriguez J, Lopez JP, Blair KJ, Yu MA, Haas M, Brumund KT, et al: EGFR kinase promotes acquisition of stem cell-like properties: a potential therapeutic target in head and neck squamous cell carcinoma stem cells. PLoS One 7: e32459, 2012.

30. Brabletz T, Jung A, Spaderna S, Hlubek F and Kirchner T: Opinion: Migrating cancer stem cells-an integrated concept of malignant tumour progression. Nat Rev Cancer 5: 744-749, 2005.

31. Brabletz T: EMT and MET in metastasis: Where are the cancer stem cells? Cancer Cell 22: 699-701, 2012.

32. Biddle A, Liang X, Gammon L, Fazil B, Harper LJ, Emich H, Costea DE and Mackenzie IC: Cancer stem cells in squamous cell carcinoma switch between two distinct phenotypes that are preferentially migratory or proliferative. Cancer Res 71: 5317-5326, 2011.

33. Liu S, Clouthier SG and Wicha MS: Role of microRNAs in the regulation of breast cancer stem cells. J Mammary Gland Biol Neoplasia 17: 15-21, 2012.

34. French R and Clarkson R: The complex nature of breast cancer stem-like cells: Heterogeneity and plasticity. J Stem Cell Res Ther S7: 009, 2012. 